\title{
Comunidad de arañas (Arachnida, Araneae) del cultivo de alfalfa (Medicago sativa) en Buenos Aires, Argentina
}

\author{
Andrea Armendano \& Alda González \\ Centro de Estudios Parasitológicos y de Vectores (CEPAVE - CONICET), 2 N$^{\circ} 584$ (1900) La Plata, Argentina, Fax \\ 054-221-4232327; aarmendano@hotmail.com, asgonzalez@cepave.com.ar
}

Recibido 15-VI-2009. Corregido 09-X-2009. Aceptado 10-XI-2009.

\begin{abstract}
Spider community (Arachnida, Araneae) of alfalfa crops (Medicago sativa) in Buenos Aires, Argentina. Over the last decades there has been an increasing interest in the use of natural enemies to control pest insects, including spiders. We studied a spider community in Argentina by sampling every two weeks during 2004-2006 in one-hectare lots. Soil stratum spiders were collected using nets and pitfall traps. A total of 6229 specimens were collected (15 families and 50 species). Seven families were found in the herbal stratum, the most abundant were Thomisidae $(\mathrm{n}=2012,32.30 \%)$, Araneidae $(\mathrm{n}=1516,24.33 \%)$ and Oxyopidae $(\mathrm{n}=604,9.70 \%)$. The soil had 14 families, mainly: Lycosidae $(n=629,10.10 \%)$ and Linyphiidae $(n=427,6.85 \%)$. Hunting spiders predominated: ambushers (32.99\%); stalkers (11.77\%) and ground-runners (10.84\%) were less common. The most abundant web building spiders were the orb weavers $(27.56 \%)$. The diversity indexes were: $\mathrm{H}^{\prime}=2.97$, $\mathrm{Dsp}=0.11$ and $\mathrm{J}=0.79$, evidencing a moderately diverse spider community with predominance of Misumenops pallidus, Oxyopes salticus, Lycosa poliostoma and L. erythrognatha. The spiders were present throughout the phenological development of the crop with abundance peaks in spring and summer. Rev. Biol. Trop. 58 (2): 757-767. Epub 2010 June 02.
\end{abstract}

Key words: araneae, alfalfa, agroecosystems, natural enemies.

Las arañas constituyen uno de los principales grupos de la fauna de artrópodos en los sistemas agrícolas, que pueden alimentarse del 40 al $50 \%$ de la biomasa disponible de insectos, teniendo un papel importante en el control biológico de las especies que provocan daños en los cultivos de importancia económica (Riechert \& Lockley 1984, Tarabaev \& Sheykin 1990, Green 1996). Investigaciones en diversos agroecosistemas han demostrado que el impacto de la depredación colectiva de las arañas contribuye a disminuir los niveles de plagas en campos de cultivos (Riechert \& Bishop 1990, Provencher \& Riechert 1994, Nyffeler et al. 1994, Riechert \& Lawrence 1997, Nyffeler 1999, Symondson et al. 2002). La estructura del hábitat es un factor determinante que mantiene distintas asociaciones de arañas y que resulta ser crítica al considerar la reducción de las poblaciones de insectos (Hatley \& MacMahon 1980, Uetz 1991, Wise 1993, Pearce et al. 2003). La complejidad estructural determina la composición de los gremios de arañas e indirectamente influye en el nivel de daño ocasionado por los herbívoros (Young \& Edwards 1990, Ysuet \& Canard 2000). Por lo tanto, la identificación de los gremios de arañas, su composición y los factores que influyen en la estructura de su comunidad, son esenciales en los estudios sobre la fauna de artrópodos de los agroecosistemas (Uetz et al. 1999, Pearce et al. 2003).

La alfalfa es un cultivo perenne, con una vida útil de cuatro a seis años con una gran cobertura foliar y que se encuentra entre los cultivos con mayor diversidad de arañas (Young 
\& Edwards 1990), conjuntamente con los de algodón y de soja. Además provee un hábitat favorable y un recurso alimentario considerable para un gran número de especies de insectos, muchos de los cuales son considerados plaga por el daño que causan al alimentarse de las hojas, tallos y raíces de las plantas (Harcourt et al. 1986, Carrasco \& Báez 2006).

En Argentina se han realizado estudios sobre las arañas de agroecosistemas, principalmente en cultivos de soja (Minervino 1996, Liljesthröm et al. 2002, Beltramo et al. 2006), pero no existen trabajos previos referidos al cultivo de alfalfa, por lo que el presente trabajo es la primera contribución al conocimiento de la composición, diversidad, riqueza, abundancia y estructura de sus gremios en un cultivo de alfalfa de la provincia de Buenos Aires.

\section{MATERIALES Y MÉTODOS}

Área de estudio: comprendió lotes de una hectárea cultivados con alfalfa, ubicados en la Estación Experimental de Gorina, partido de La Plata $\left(34^{\circ} 53^{\prime}\right.$ S- S $\left.58^{\circ} 05^{\prime}, \mathrm{W}\right)$, provincia de Buenos Aires, Argentina. El cultivo fue mantenido de acuerdo a las prácticas convencionales de manejo agrícola. Se realizó siembra directa con una única aplicación de herbicidas preemergentes y no se aplicaron insecticidas. El cultivo estuvo rodeado por zonas adyacentes de vegetación natural, donde se encontró una gran variedad de especies de Compositae, Graminea y Cruciferae, siendo las dominantes: Carduus acanthoides Linneo, Bromus unioloides Kunth y Raphanus sativus (Linneo). Esta Estación se encuentra dentro del área de clima templado con una temperatura media anual de $16.3^{\circ} \mathrm{C}$ y una precipitación media anual de $993.7 \mathrm{~mm}$.

Muestreo de arañas: se realizó quincenalmente, entre 2004 y 2006. La recolección de los ejemplares abarcó dos estratos, el suelo donde se colocaron trampas de caída (pitfalls) de $6.5 \times 12 \mathrm{~cm}$ (diámetro $\mathrm{x}$ profundidad) con etilenglicol y agua como solución conservante (3:1) y el herbáceo donde se utilizó red de arrastre de $38 \mathrm{~cm}$ de diámetro. En cada muestreo se distribuyeron 20 trampas de caída en cuatro transectos lineales paralelos ubicados en dirección N-S. En cada transecto se colocaron cinco trampas de caída cada $20 \mathrm{~m}$ y en esos puntos se procedió a la recolección de 20 muestras con red de arrastre sobre el estrato herbáceo, consistiendo cada una en seis golpes de red sobre la vegetación del cultivo. Las arañas capturadas se fijaron con alcohol $70 \%$ e identificaron a través de claves de arañas argentinas y de acuerdo a la bibliografía taxonómica (Platnick 2009). Cuando no fue posible la identificación específica, los ejemplares fueron separados en grupos de especímenes morfológicamente idénticos (morfoespecies), a nivel de género o familia. Se estableció el sexo y estado de desarrollo de cada ejemplar. Al finalizar el estudio los ejemplares fueron depositados en el Laboratorio Aracnológico del Centro de Estudios Parasitológicos y de Vectores (Universidad Nacional de La Plata).

Análisis estadísticos: se utilizó el programa EstiMateS (Colwell 2006), se calculó la riqueza de especies (S) y la diversidad mediante los índices de Shannon-Wienner $\left(\mathrm{H}^{\prime}\right)$, de Simpson (D) y de Equidad de Pielou (J) y los estimadores Chao 1, Chao 2, Jacknife de primer y segundo orden (Colwell \& Coddington 1994, Moreno 2001). Para el estudio de la estructura de gremios de la comunidad de arañas de alfalfa se consideró la clasificación de Uetz et al. 1999.

\section{RESULTADOS}

Composición taxonómica y estructura de gremios: se recolectaron un total de 6229 arañas, pertenecientes a 15 familias y 50 especies. En la vegetación del cultivo se capturaron 4681 ejemplares, representantes de siete familias y en el estrato del suelo se obtuvieron 1548 ejemplares, representantes de 14 familias. Las más abundantes en el estrato herbáceo fueron: Thomisidae $(\mathrm{n}=2012,32.30 \%)$, Araneidae $(\mathrm{n}=1$ $516,24.33 \%)$ y Oxyopidae $(\mathrm{n}=604,9.70 \%)$ y para el estrato del suelo: Lycosidae $(n=629$, $10.10 \%)$ y Linyphiidae $(\mathrm{n}=427,6.85 \%)$. El 
resto de las familias no superaron el $6 \%$ de abundancia (Cuadro 1).

Las familias recolectadas se agruparon en nueve gremios. Predominaron las arañas cazadoras, representadas por: las cazadoras por emboscada (32.99\%), las cazadoras al acecho $(11.77 \%)$ y las cazadoras corredoras de suelo $(10.84 \%)$. Entre las tejedoras, las

\section{CUADRO 1}

Composición, abundancia y riqueza específica de las familias capturadas en el cultivo de alfalfa agrupadas por gremios (Buenos Aires, Argentina)

TABLE 1

Composition, abundance and specific richness of spider families captured in alfalfa crops by guild (Buenos Aires, Argentina)

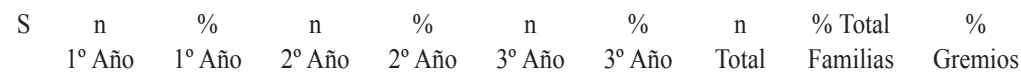

Tejedoras telas sábanas

Amaurobiidae

Hahniidae

$\begin{array}{ccccccccc}2 & 10 & 0.49 & 29 & 1.40 & 22 & 1.04 & 61 & 0.98 \\ 1 & 66 & 3.24 & 42 & 2.02 & 57 & 2.69 & 165 & 2.65\end{array}$

Tejedoras telas orbiculares

Araneidae

Tetragnathidae

Tejedoras telas irregulares

Theridiidae

Tejedoras errantes telas sábanas

Linyphiidae

$$
13 \quad 623
$$$$
30.63
$$$$
167
$$

$$
2 \quad 11
$$

$$
0.54
$$$$
467
$$

56

22.51

426

20.09

1516

$\begin{array}{llll}2.70 & 78 & 3.68 & 201\end{array}$

27.56

0.55

$8 \quad 120 \quad 5.90$

Trap doors

Actinopodidae

$\begin{array}{llll}1 & 1 & 0.05 & 2\end{array}$

0.10

157

7.41

427

6.85

Cazadoras por emboscada

Philodromidae

Thomisidae

$\begin{array}{ccccccccc}2 & 9 & 0.44 & 18 & 0.87 & 16 & 0.75 & 43 & 0.69 \\ 6 & 612 & 30.09 & 687 & 33.11 & 713 & 33.63 & 2012 & 32.30\end{array}$

Cazadoras corredoras suelo

Coriniidae

Gnaphosidae

Lycosidae

$\begin{array}{ccc}2 & 5 & 0.25 \\ 1 & 2 & 0.10 \\ 2 & 176 & 8.65\end{array}$

Cazadoras corredoras follaje

Anyphaenidae

$3 \quad 113 \quad 5.56$

.25

8$$
0.39
$$$$
0.48
$$

9

$$
9
$$

$$
0.42
$$$$
0.57
$$$$
22
$$$$
24
$$$$
11.56 \quad 629
$$

0.35

208

10.02

245

629

Cazadoras al acecho

Oxyopidae

Salticidae

$1 \quad 180$

8.85

209
51

10.07

215

10.14

604

604

9.70

Total

$50 \quad 2034$

100

2075

2.46

39

1.84 
más abundantes fueron las que construyen telas orbiculares $(27.56 \%)$. El resto de los gremios no superó el 7\%. El gremio que totalizó el mayor número de individuos fue el de las cazadoras por emboscada $(n=2055)$. Le siguió el de las tejedoras orbiculares $(n=1$ 717), que presentó además la mayor riqueza de especies $(\mathrm{S}=14)$. El resto de los gremios presentaron números de individuos inferiores a los 750 y no superaron las 8 especies (Cuadro 1). Todos los ejemplares capturados pertenecen al Infraorden Araneomorphae, salvo cuatro ejemplares machos de la familia Actinopodidae pertenecientes a Mygalomorphae ( $\mathrm{n}=4,0.06 \%$ ) (Cuadro 2). Del total de 6 229 arañas registradas, 3735 (59.96\%) fueron juveniles. Entre los adultos se capturaron una mayor cantidad de machos $(\mathrm{n}=1720,69 \%)$ que de hembras ( $n=773,31 \%)$, especialmente con trampas de caída, los machos mayoritariamente de las especies: Lycosa poliostoma (Koch, 1847), Lycosa erythrognatha (Lucas, 1836) (Lycosidae) y Glenognatha lacteovittata (Mello-Leitão, 1944) (Tetragnathidae).

\section{CUADRO 2}

Familias y especies/morfoespecies de las arañas asociadas al cultivo de alfalfa (Buenos Aires, Argentina). Método de colecta (A) Red de arrastre (B) Trampas de caida

TABLE 2

Families and species/morphospecies of spidersassociated with alfalfa crops (Buenos Aires, Argentina)

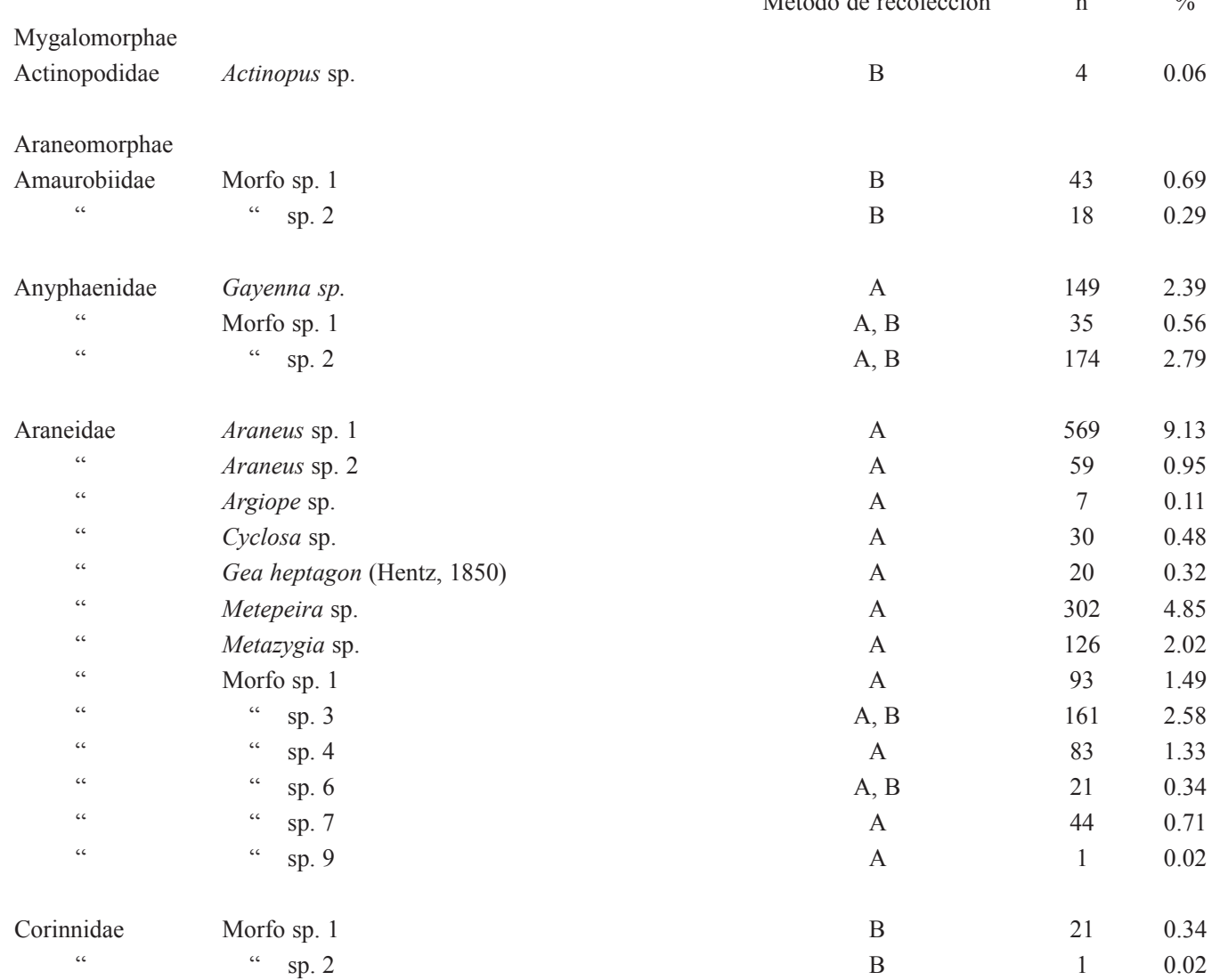


CUADRO 2 (Continuación)

Familias y especies/morfoespecies de las arañas asociadas al cultivo de alfalfa (Buenos Aires, Argentina). Método de colecta (A) Red de arrastre (B) Trampas de caída

TABLE 2 (Continued)

Families and species/morphospecies of spidersassociated with alfalfa crops (Buenos Aires, Argentina)

\begin{tabular}{|c|c|c|c|c|}
\hline & & & & \\
\hline Gnaphosidae & Morfo sp. 1 & B & 24 & 0.39 \\
\hline Hahniidae & Hahnia sp. & $\mathrm{B}$ & 165 & 2.65 \\
\hline Lycosidae & Lycosa poliostoma (Koch, 1847) & $\mathrm{B}$ & 336 & 5.39 \\
\hline “ & Lycosa erythrognatha (Lucas, 1836) & $\mathrm{B}$ & 293 & 4.70 \\
\hline Linyphiidae & Meioneta sp. & A, B & 200 & 3.21 \\
\hline “ & Morfo sp. 1 & A, B & 120 & 1.93 \\
\hline “ & “ $\quad$ sp. 2 & $\mathrm{~B}$ & 9 & 0.14 \\
\hline “ & “ sp. 3 & A, B & 38 & 0.61 \\
\hline “ & “ sp. 4 & A, B & 39 & 0.63 \\
\hline “ & Erigoninae & $\mathrm{B}$ & 14 & 0.22 \\
\hline “ & “ $\quad$ sp. 6 & $\mathrm{~B}$ & 2 & 0.03 \\
\hline “ & “ $\quad$ sp. 8 & $\mathrm{~B}$ & 5 & 0.08 \\
\hline Oxyopidae & Oxyopes salticus (Hentz, 1845) & A & 604 & 9.70 \\
\hline Philodromidae & Morfo sp. 1 & A & 28 & 0.45 \\
\hline “ & “ sp. 2 & A & 15 & 0.24 \\
\hline Salticidae & Dendryphantinae & A, B & 68 & 1.09 \\
\hline “ & Morfo sp. 1 & A, B & 42 & 0.67 \\
\hline “ & “ $\quad$ sp. 2 & A, B & 12 & 0.19 \\
\hline “ & “ sp. 4 & $\mathrm{~A}, \mathrm{~B}$ & 6 & 0.10 \\
\hline “ & “ sp. 6 & $\mathrm{~A}, \mathrm{~B}$ & 1 & 0.02 \\
\hline Tetragnathidae & Glenognatha lacteovittata (Mello-Leitão, 1944) & B & 201 & 3.23 \\
\hline Theridiidae & Parasteatoda tepidariorum (C. L. Koch, 1841) & $\mathrm{A}, \mathrm{B}$ & 29 & 0.47 \\
\hline & Theridion sp. & A & 5 & 0.08 \\
\hline Thomisidae & Misumenops pallidus (Keyserling, 1880) & A & 1738 & 27.90 \\
\hline “ & Misumenops sp. & A & 149 & 2.39 \\
\hline “ & Misumena sp. & A & 55 & 0.88 \\
\hline “ & Morfo sp. 1 & A & 36 & 0.58 \\
\hline “ & “ sp. 2 & A & 13 & 0.21 \\
\hline “ & “ sp. 3 & A & 21 & 0.34 \\
\hline $\mathrm{N}$ & & & 6229 & 100 \\
\hline
\end{tabular}

Collection techniques (A) Sweeping net (B) Pitfall. 
Composición específica, riqueza y diversidad de especies: se registró una riqueza específica $(S)=50$, con 36 especies en el estrato herbáceo y 14 en el suelo (índice de Jaccard=0.44). La especie más abundante del estrato herbáceo fue Misumenops pallidus (Keyserling, 1880) ( $\mathrm{n}=1$ 738) y Misumenops sp. $(\mathrm{n}=149)$ (Thomisidae). También fueron abundantes: Oxyopes salticus Hentz, 1845 $(\mathrm{n}=604)$ (Oxyopidae) (nueva cita para Argentina aunque figura en el trabajo no publicado de Cristian Grismado 2007), Araneus sp. 1 $(\mathrm{n}=569)$, Metepeira sp. $(\mathrm{n}=302)$, Metazygia sp. $(\mathrm{n}=126)$ y morfoespecie $3(\mathrm{n}=161)$ (Araneidae), Gayenna sp. (n=149) y morfoespecie $2(n=174)$ (Anyphaenidae). En el estrato del suelo las especies más abundante fueron: L. poliostoma $(\mathrm{n}=336)$ y L. erythrognatha ( $\mathrm{n}=293)$ (Lycosidae), seguida de Meioneta $\mathrm{sp}$. $(\mathrm{n}=200)$ (Linyphiidae), Glenognatha lacteovittata $(\mathrm{n}=201)$ (Tetragnathidae) y Hahnia sp. $(\mathrm{n}=165)$ (Hahniidae) (Cuadro 2). Las familias Araneidae y Linyphiidae fueron las de mayor riqueza específica $(\mathrm{S}=13$ y $\mathrm{S}=8$ respectivamente). De las 50 especies registradas tres fueron "singletons" (especies representadas por un único individuo en la muestra) (6\% de todas las especies) y solo una "doubletons" (especies representadas por dos individuos en la muestra) (2\%). Solo tres especies estuvieron representadas por más de 550 especímenes
(Araneus sp. 1, O. salticus y M. pallidus) con un $46.7 \%$ del total de ejemplares capturados (Cuadro 2). Los valores de los índices de diversidad de Shannon-Wiener, de Simpson y de equidad obtenidos fueron: $\mathrm{H}^{\prime}=2.97$, $\mathrm{Dsp}=0.11$ y $\mathrm{J}=0.79$ (Cuadro 3). Considerando el total de arañas capturadas en el cultivo de alfalfa los valores de los estimadores oscilaron entre 50 (Chao 1) y 59 (Jacknife de primer y de segundo orden) especies, para una riqueza observada total $\left(\mathrm{S}_{\mathrm{obs}}\right)$ de 50 especies (Cuadro 3). Entre los estratos los valores fueron de 35 (Chao 1) a 40 (Jacknife de primer y de segundo orden) para la vegetación $\left(\mathrm{S}_{\mathrm{obs}}=34\right)$ y entre 48 (Jacknife de primer y de segundo orden) y 58 (Chao 1) para el suelo $\left(\mathrm{S}_{\mathrm{obs}}=40\right)$.

Análisis temporal: se encontraron arañas a lo largo del desarrollo fenológico del cultivo. En el estrato herbáceo el pico de mayor abundancia se registró a finales de la primavera y a principios del verano (noviembre y diciembre). El mayor número de arañas registradas correspondió al mes de noviembre $(\mathrm{n}=788$, $16.83 \%$ ) (Fig. 1) donde predominaron las familias Thomisidae (M. pallidus), Araneidae (Araneus sp., Metepeira sp.) y Oxyopidae ( $O$. salticus), que constituyeron el $66.33 \%$ del total de arañas capturadas en la vegetación. El registro del menor número de arañas correspondió al mes de junio $(n=196,4.19 \%)$. En el estrato

CUADRO 3

Número de individuos, número de especies e índices de diversidad de las arañas del cultivo de alfalfa (Buenos Aires, Argentina)

TABLE 3

Numbers of individuals, numbers of species and diversity indexes of spiders in alfalfa crops (Buenos Aires, Argentina)

$\begin{array}{lccc} & \text { Vegetación } & \text { Suelo } & \text { Total } \\ \mathrm{S} & 34 & 40 & 50 \\ \mathrm{n} & 4681 & 1548 & 6229 \\ \mathrm{H}^{\prime} & 2.36 & 2.51 & 2.97 \\ \mathrm{Dsp} & 0.17 & 0.12 & 0.11 \\ \mathrm{~J} & 0.76 & 0.78 & 0.79 \\ \text { Chao 1 } & 35 \pm 1.05 & 58 \pm 1.11 & 50 \pm 2.03 \\ \text { Chao 2 } & 38.02 \pm 0.94 & 45.33 \pm 1.04 & 55.82 \pm 1.51 \\ \text { Jacknife } 1^{\circ} \text { orden } & 40.5 \pm 1.63 & 48 \pm 1.87 & 59.5 \pm 2.45 \\ \text { Jacknife } 2^{\circ} \text { orden } & 40.5 \pm 1.14 & 48 \pm 1.43 & 59.5 \pm 1.86\end{array}$



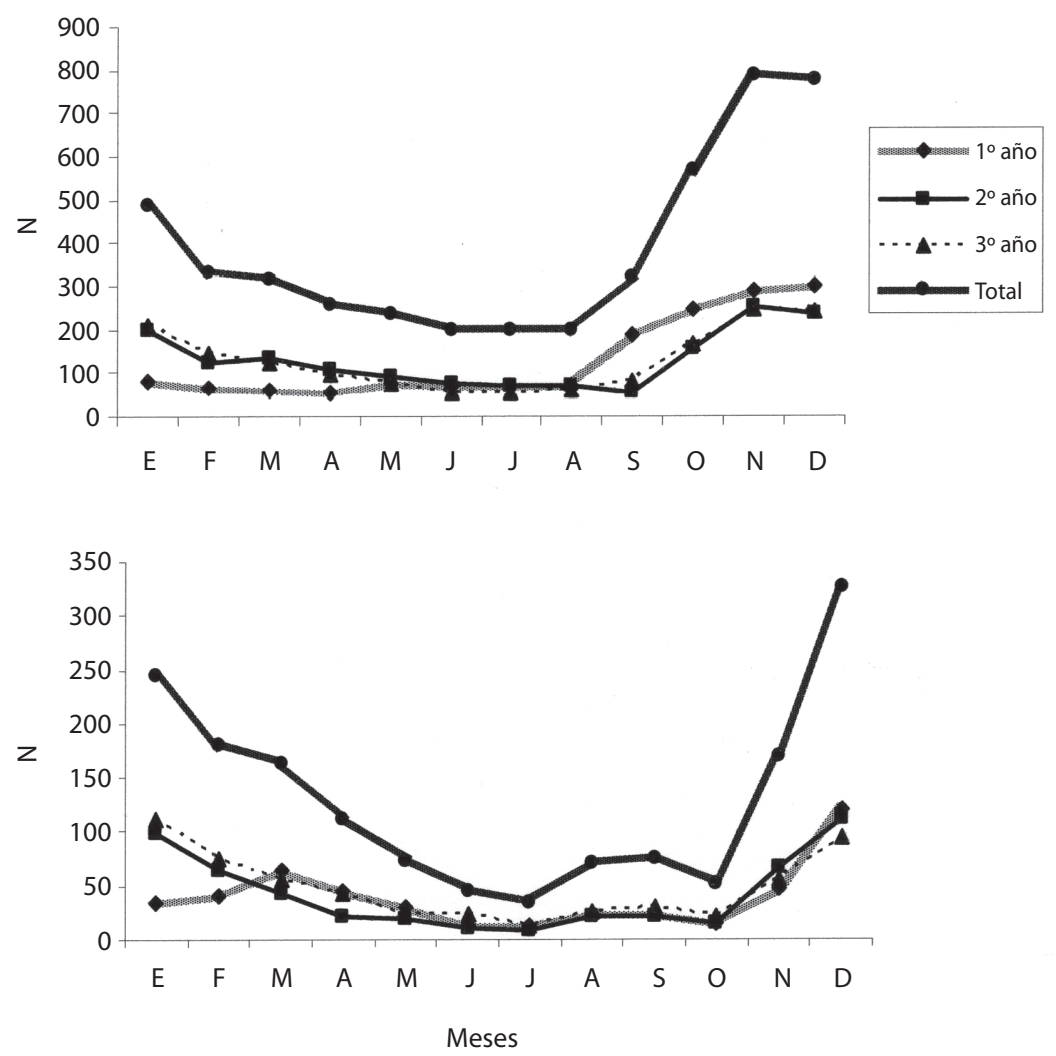

Fig. 1. Abundancia del total arañas capturadas durante el desarrollo fenológico del cultivo de alfalfa (Buenos Aires, Argentina). (A) Estrato herbáceo (B) Estrato del suelo.

Fig.1. Total spiders abundance during the fenological development of alfalfa crops (Buenos Aires, Argentina). (A) Herbal stratum (B) Soil stratum.

del suelo las familias Lycosidae (L. poliostoma, L. erythrognatha), Linyphiidae (Meioneta sp.) y Tetragnathidae ( $G$. lacteovittata), que representaron el $20.18 \%$, fueron las más abundantes especialmente durante el verano (diciembre) $(\mathrm{n}=327,21.12 \%)$. El menor número de arañas se registró en el mes de julio $(n=34,2.20 \%)$.

\section{DISCUSIÓN}

Desde el punto de vista taxonómico las 15 familias registradas en el cultivo de alfalfa representaron sólo la cuarta parte del total de familias citadas para Argentina (Pikelin \& Schiapelli 1963, Platnick 2009), resultados esperables por tratarse de un monocultivo, caracterizado por la disminución de la biodiversidad en general, incluidos los enemigos naturales de plagas agrícolas (Altieri 1995). De las 15 familias registradas, cinco representaron el $65 \%$ del total capturado (Araneidae, Thomisidae, Lycosidae, Oxyopidae y Linyphiidae). Estos resultados coinciden con Young y Edwards (1990) donde Araneidae, Linyphiidae, Lycosidae, Salticidae y Theridiidae constituyeron el $61 \%$ del total registrado para cultivos norteamericanos, siendo a su vez las familias: Araneidae, Thomisidae y Salticidae las que contribuyeron con mayor número de especies. Sin embargo, los resultados de la composición taxonómica registrada 
para el cultivo de alfalfa difieren de la de los sistemas agrícolas europeos (Nyffeler \& Sunderland 2003), donde si bien aparecen distintas familias (Linyphiidae, Lycosidae, Araneidae, Tetragnathidae y Theridiidae), la fauna está ampliamente dominada por Linyphiidae (9399\% del total de arañas), siendo las arañas cazadoras las menos representadas (14.2\%) (Luczak 1979, Nyffeler \& Benz 1987).

La familia de arañas más frecuente en el estrato herbáceo del cultivo de alfalfa fue Thomisidae representada por la especie $M$. pallidus. Estas arañas son deambuladoras, que cazan a sus presas al acecho, ya que esperan a los insectos que se alimentan de polen y néctar, entre las a flores e inflorescencias a las que se asocian. En el estrato del suelo predominó la familia Lycosidae, representada por L. poliostoma y L. erythrognatha. Estas arañas viven en madrigueras que construyen ellas mismas en el sustrato, las que tapizan con seda. Son de hábitos nocturnos, se desplazan sobre la superficie del suelo y bajo la hojarasca en busca de sus presas. En ambos casos se trata de arañas cazadoras, lo que coincide con Young \& Lockley (1985) y Young \& Edwards (1990) que observaron que más del $50 \%$ de las arañas recolectadas en las zonas cultivadas son cazadoras, entre las cuales $O$. salticus constituye la especie más comúnmente hallada en cultivos extensivos de soja y algodón de América del Norte. Registros similares se han obtenido en nuestro país en cultivos de soja, en la zona central de la Provincia de Santa Fe, donde la especie más abundante del estrato herbáceo del cultivo y zonas aledañas fue $O$. salticus (Beltramo et al. 2006). En zonas secas Oxyopidae sería reemplazada por Thomisidae, como en el oeste de Texas y Arizona donde predominan Misumenops sp. (Young \& Edwards 1990). M. pallidus ha sido registrada en otros agroecosistemas sudamericanos, tal el caso de estudios realizados en cultivos de sorgo en Brasil donde fue la especie más abundante (25.6\%) (Campos et al. 1999). Otros estudios realizados en arroz y cítricos en Brasil y cacao en Méjico muestran un predominio de araneidos pero también se encuentran registros de oxyópidos y tomísidos pero en menor porcentaje (Ott et al. 2007, Pérez de la Cruz et al. 2007, Rodrigues et al. 2008).

Según Nyffeler \& Sunderland (2003) existen diferencias en la riqueza específica de las arañas encontradas en distintos cultivos, pero la proporción de especies de arañas dentro de los gremios individuales es relativamente constante. En promedio el $16.9 \%$ de todas las especies de arañas de las comunidades agrícolas fueron cazadoras al acecho. Las especies cazadoras por emboscada, cazadoras corredoras de suelo, corredoras sobre la vegetación y tejedoras tipo sábana constituyeron un porcentaje relativamente fijo de la comunidad de arañas de agroecosistemas (Uetz et al. 1999). En la alfalfa también predominaron las arañas cazadoras, pero a diferencia de los registros de Uetz et al. (1999) se destacaron las arañas cazadoras por emboscadas. Sin embargo la gran cobertura foliar y la complejidad de la estructura de la vegetación de este cultivo, parece tener un efecto directo sobre el emplazamiento de las telas y una mayor disponibilidad de sustratos donde fijarlas (Rypstra 1985, Samu \& Szinetár 2002), lo que queda evidenciado en la gran densidad de arañas pertenecientes a los gremio de tejedoras de telas orbiculares y de telas tipo sábana. Resultados semejantes se registraron en nuestro país en cultivos de soja, donde predominaron en el estrato herbáceo las arañas pertenecientes a los gremios I (Thomisidae, Anyphaenidae y Philodromidae) y III (Araneidae excepto el género Alpaida) y en el estrato del suelo las arañas del gremio V (Lycosidae y Gnaphosidae) (Minervino 1996, Liljesthröm et al. 2002).

Desde el punto de vista temporal el registro de arañas durante todo el año indica que este tipo de cultivo funciona como un hábitat estable, donde la permanente cobertura del suelo favorece la disponibilidad de presas y la provisión de refugios, sirviendo como sitios de preferencia para el establecimiento de las comunidades de arañas (Duffey 1978, Descender et al. 1989). Se registra además un escaso número de taxones representados por singletons y doubletons, lo que coincide 
con Young y Edwards (1990) y se evidencia a través de los valores de los índices de diversidad que muestran una comunidad de arañas moderadamente diversa, con predominio de algunas especies (M. pallidus, O. salticus y Araneus sp. 1, L. poliostoma, L. erythrognatha, Meioneta sp., Glenognatha lacteovittata y Hahnia sp.), si se considera que en la mayoría de los casos los valores para estos índices se ubican entre 1.5 y 3.5 , raramente excediendo 4.5 (Magurran 1988).

En Argentina, si bien existe poca información respecto a las comunidades de arañas de agroecosistemas, cabe mencionar los estudios realizados en el cultivo de soja no transgénica (Minervino 1996, Liljesthröm et al. 2002, Beltramo et al. 2006) donde se obtuvieron resultados similares en cuanto a la composición taxonómica y diversidad específica. En éstos la riqueza específica total fue de 37 especies, agrupadas en 13 familias. Las familias dominantes fueron Thomisidae (M. pallidus) en el estrato herbáceo y Lycosidae en estrato del suelo. Teniendo en cuenta la bibliografía aracnológica actual, el presente trabajo constituye el primer estudio sobre la araneofauna del cultivo de alfalfa en este país. Es necesario continuar los estudios en otros sistemas agrícolas para lograr un panorama más amplio y comparaciones objetivas referidas a la comunidad de arañas en estos sistemas particulares.

\section{RESUMEN}

En las últimas décadas se ha dado un interés creciente en el uso de enemigos naturales para controlar plagas de insectos, como arañas. Se estudió una comunidad de arañas en Argentina mediante un muestreo cada dos semanas durante el periodo 2004-2006 en lotes de una hectárea. En el estrato del suelo las arañas fueron colectadas con redes de arrastre y trampas de caída. Se recolecto un total de 6 229 ejemplares (15 familias y 50 especies). Siete familias se encuentran en el estrato herbáceo, las más abundantes fueron: Thomisidae $(\mathrm{n}=2$ 012, 32.30\%), Araneidae $(\mathrm{n}=1$ $516,24.33 \%)$ y Oxyopidae $(\mathrm{n}=604,9.70 \%)$. El suelo habían 14 familias, principalmente: Lycosidae $(n=629$, $10.10 \%)$ y Linyphiidae $(\mathrm{n}=427,6.85 \%)$. Predominaron las arañas cazadoras: por emboscadas (32.99\%), al acecho (11.77\%), corredoras de suelo (10.84\%) y tejedoras orbiculares $(27.56 \%)$. Los índices de diversidad fueron: $\mathrm{H}^{\prime}=2.97$, $\mathrm{Dsp}=0.11$ y $\mathrm{J}=0.79$, evidenciando una comunidad de arañas moderadamente diversa, con predominio de Misumenops pallidus, Oxyopes salticus, Lycosa poliostoma and $L$. erythrognatha. Las arañas estuvieron presentes durante el desarrollo fenológico del cultivo con picos de abundancia en primavera y verano.

Palabras clave: araneae, alfalfa, agroecosistemas, enemigos naturales.

\section{REFERENCIAS}

Altieri, M.A. 1995. Agroecology: the Science of Sustainable Agriculture. Westview, Boulder, Colorado, EEUU.

Beltramo, J., I. Berolaccini \& A. González. 2006. Spiders of soybean crops in Santa Fe Province,

Argentina: Influence of surrounding spontaneus vegetation on lot colonization. Braz. J. Biol. 66: 29-41.

Campos, A.R., E.B. Filho, F.M. Lara \& I.M.P. Rinaldi. 1999. Composição da Artropodofauna Entomófoga Associada a Diferentes Genótipos de Sorgo Granífero no Cerrado do Sudeste do Brasil. An. Soc. Entomol. Brasil 28: 703-714.

Carrasco, N. \& A. Báez. 2006. Trigo: manual de campo. INTA EEA Integrada Barrow Tres Arroyos, Buenos Aires, Argentina.

Colwell, R. \& J.A. Coddington. 1994. Estimating terrestrial biodiversity through extrapolation. Phil. Trans. R. Soc. Lond. 345: 102-118.

Colwell, R. 2006. EstiMateS; statistical estimation of species richness and shared species from simples. Version 8.0. Department of Ecology and Evolutionary Biology, University of Connecticut Storrs. Connecticut, EEUU.

Descender, K., M. Alderweireldt \& M. Pollet. 1989. Field edges and their importance for polyphagous predatory arthropods. Med. Fac. Landbouww. Rijksuniv. Gent. 54: 823-833.

Duffey, E. 1978. Ecological strategies in spidersincluding some characteristics of spicies in pioneer and nature habitats. Symp. Zool. Soc. London 42: 109-123.

Green, J. 1996. Spiders in biological control- An Australian perspective. Rev. Suisse Zool. Vol. hors série: 245-253.

Grismado, C. 2007. Comunidades de arañas de la Reserva Natural Otamendi, Provincia de Buenos Aires. Riqueza específica y Diversidad. Departamento de Ciencias Biológicas, Universidad CAECE. Buenos Aires, Argentina. 
Harcourt, D.C., J.R. Aragón \& R. González. 1986. Plagas de la alfalfa. In C. Bariggi, B.L. Marble, C. D. Itria \& J.M. Brun. Investigación, tecnología y producción de alfalfa. Colección Científica del INTA, Tomo XXII Buenos Aires, Argentina.

Hatley, C. \& J.A. MacMahon. 1980. Spider Community Organization: Seasonal Variation and the Role of Vegetation Architecture. Environ. Entomol. 9: 632-639.

Liljesthröm, G, E. Minervino, D. Castro \& A. González. 2002. La Comunidad de Arañas del Cultivo de

Soja en la Provincia de Buenos Aires, Argentina. Neotrop. Entomol. 31: 197-209.

Luczak, J. 1979. Spiders in agrocoenoses. Pol. Ecol. Stud. 1: $151-200$

Magurran, A. 1988. Ecological diversity and its measurement. Princeton University, Nueva Jersey, EEUU.

Minervino, E. 1996. Estudio biológico y ecobiológico de arañas depredadoras de plagas de soja. Tesis de Doctorado, Universidad de La Plata, La Plata, Argentina.

Moreno, C.E. 2001. Métodos para medir la biodiversidad M \& T, Manuales y Tesis SEA. Vol. 1. Zaragoza, España.

Nyffeler, M. \& G. Benz. 1987. Spiders in natural pest control: a review. Appl. Entomol. 103: 321-339.

Nyffeler, M. 1999. Prey selection of spiders in the field. J. Arachnol. 27: 317-324.

Nyffeler, M., W.L. Sterling \& D.A. Dean. 1994. Insectivorous activities of spiders in United States field crops. J. Appl. Entomol. 118: 113-128.

Nyffeler, M. \& K. Sunderland. 2003. Composition, abundance and pest control potential of spider communities in agroecosystems: a comparison of European and US studies. Agr. Ecosyst. Environ. 95: 579-612.

Ott, A., R. Ott \& V.R.S. Wolff. 2007. Araneofauna de pomares de laranja Valência nos Vales do Caí e Taquari, Rio Grande do Sul, Brasil. Iheringia, Sér. Zool. 97: 321-327.
Pearce, J.L., L.A. Venier, G. Eccles, J. Pedlar \& D. McKenney. 2003. Influence of habitat and microhabitat on epigeal spider (Araneae) assemblages in four stand types. Biodivers. Conserv. 13: 1305-1334.

Pérez-de la Cruz M., S. Sánchez-Soto, C.F. Ortíz-García, R. Zapata-Mata \& A. de la Cruz-Pérez. 2007. Diversidad de Insectos Capturados por Arañas Tejedoras (Arachnida: Araneae) en el Agroecosistema Cacao en Tabasco, México. Neotrop. Entomol. 36: 90-101.

Pikelin, B.S.G. de \& R.D. Schiapelli. 1963. Llave para la determinación de las familias de arañas argentinas. Physis 24: 43-72.

Provencher, L. \& S.E. Riechert. 1994. Model and field test of prey control effects by spider assemblages. Environ. Entomol. 23: 1-17.

Riechert, S.E. \& T. Lockley. 1984. Spiders as biological control agents. Ann. Rev. Entomol. 29: 299-320.

Riechert, S.E. \& L. Bishop. 1990. Prey control by an assemblage of generalist predators: spiders in garden test system. Ecology 71: 1441-1450.

Riechert, S.E. \& K. Lawrence. 1997. Test for predation effects of single versus multiple species of generalist predators: Spiders and their insect prey. Entomol. Exp. Appl. 4: 147-155.

Rodrigues, E.N.L., M.S. Mendonça Jr. \& R. Ott. 2008. Fauna de aranhas (Arachnida, Araneae) em diferentes estágios do cultivo do arroz irrigado em Cachoeirinha, RS, Brasil. Iheringia Sér. Zool. 98: 362-371.

Rypstra, A. 1985. Agregations of Nephila clavipes (L.) (Araneae: Araneidae) in relation to prey availability. J. Arachnol. 13: 71-78.

Samu, F. \& C. Szinetár. 2002. On the nature of agrobiont spiders. J. Arachnol. 30: 389-402.

Symondson, W.O.C., K.D. Sunderland \& M.H. Greenstone. 2002. Can generalist predators be effective biocontrol agents? Annu. Rev. Entomol. 47: 561-594.

Tarabaev, C.K. \& A.A. Sheykin. 1990. Spiders as predators in apple-tree crows in south-eastern in Kasakhstan. Acta Zool. Fennica 190: 363-366. 
Uetz, G. 1991. Habitat structure and spider foraging. In S. Bell, E. McCoy \& H. Mushinsky. Habitat structure: The physical arragment of obkects in space. Chapman \& Hall, Londres, Reino Unido.

Uetz, G.W., J. Halaj \& A.B. Cady. 1999. Guild structure of spiders in major crops. J. Arachnol. 27: 270-280.

Wise, D.H. 1993. Spiders in ecological webs. Cambridge University, Cambridge, EEUU.

Ysuet, F. \& A. Canard. 2000. Spider Biodiversity in connection with the vegetation structure and the foliage orientation of hedges. J. Arachnol. 28: 107-114.
Young, O.P. \& T.C. Lockley. 1985. The striped lynx spider, Oxyopes salticus (Araneae: Oxyopidae), in agroecosystems. Entomophaga 30: 329-346.

Young, O.P. \& G.B. Edwards. 1990. Spiders in United States field crops and their potential effect on crop pests. J. Arachnol. 18: 1-27.

\section{REFERENCIAS DE INTERNET}

Platnick, N.I. 2009. The World Spider Catalog, Version 10.0. American Museum of Natural History (Consultado 23 enero 2009, www.research.amnh.org/entomology/spiders/catalog/index.html). 
